\title{
Effectiveness of Teaching That can Promote the Data Modeling Ability of Junior High School Students
}

\author{
Jeng-Fung Hung, Chen-Heng Ko, Wei-Ming Yang \\ National Kaohsiung Normal University, Kaohsiung, Taiwan
}

\begin{abstract}
This study aimed at designing teaching model that can promote students' data modeling ability and investigating its effectiveness in enhancing students' data modeling ability. In this study, quasi-experimental design was adopted. Forty-six students in the experiment group were conducted with data modeling teaching, while 51 students in the control group were conducted with lecture instruction through textbook. The teaching content was Newton's second law of motion. Meanwhile, three sessions of teaching activities were conducted on both groups. Before teaching treatment, test of mechanical concept was implemented first, and after finishing the teaching activity, "Force and Motion” data modeling ability test and "Force and Motion” achievement test were implemented again. In this study, test of mechanical concept before teaching treatment was used as covariate to conduct statistical analysis with the analysis of covariance (ANCOVA). In addition, in order to understand the learning problems that might exist in the students during the data modeling process, this study used "average correct answering rate lower than $65 \%$ on the test” as judgment standard for not achieving the learning. From the research result, it was found that: 1 . After teaching, the performance in the experiment group in the data modeling ability test was superior to that in the control group $(p<0.05)$; 2 . The students' performance of achievement test in the experiment group and the control group did not reach significance difference $(p>0.05)$; 3 . The students in the experiment group showed the same learning problems in the performance of explaining data model as the students in the control group; and 4. Students in the control group showed learning problem in the performance of organizing data model, too.
\end{abstract}

Keywords: data modeling, Force and Motion, virtual experiment

\section{Introduction}

This is a data-driven society, when you read newspaper carefully, you can see all kinds of figures, tables, drawings, and other data symbol to be explained, along with the acquisition of big data. People can get involved with democratic dialogue and public decision (English, 2011), and participated in the investigation of science and technology related topics. "Data" meant things that can be recorded, analyzed, and re-organized, and it was displayed in a quantified way to facilitate arrangement and analysis, so that people can use it to

Jeng-Fung Hung, Ph.D., professor, Graduate Institute of Science Education and Environmental Education, National Kaohsiung Normal University.

Chen-Heng Ko, Ph.D. student, Graduate Institute of Science Education and Environmental Education, National Kaohsiung Normal University.

Wei-Ming Yang, M.S. student, Graduate Institute of Science Education and Environmental Education, National Kaohsiung Normal University. 
symbolize the look of natural world. Lehrer and Schauble (2000) and Lehrer and Lesh (2003) pointed out that this was a data modeling process and it started from exploration and investigation on meaningful things to decide what attributes were something worthy of attention in the phenomenon. Next, these attributes were measured and the measured data were organized, structuralized, visualized, and symbolized. Then, a model for the relationship of these attributes was constructed.

The purpose of data modeling organization was to symbolize data and let data become understandable. If students' attention can be put on the data and the data can be analyzed, then they can understand the data well. Firstly, students needed to understand that data were constructed to answer the question. What the problem was will decide information type to be collected, data-coding, and structured (National Research Council [NRC], 2008). Secondly, students needed to know that data were abstract, because they represented observation on specific thing. Data can take many tables, for example, the distance of one straight line can be represented by many standard units, dynamic image record can represent observation on people's interaction, or the reading on temperature meter can represent sense of heat. Thirdly, students needed to understand the concept of variable, including independent variable, dependent variable, and control variable, and the concept of measurement, for example, the use of quantification (measurement), quality (observation) and ruler, and type and continuous variable (Organisation for Economic Cooperation and Development [OECD], 2013). Furthermore, data did not possess structure originally, on the contrary, a structure must be added on the data. The scientists used the selection of category to describe and organize data, so as to reinforce the structure in data. In addition, data are symbolized in all kinds of methods, so as to see, understand, or communicate different faces of the phenomenon to be studied. An important learning target to the students was to understand the attributes of different types displayed by data.

\section{Literature Review}

There were many different types of symbols to be used for data display, including tables, all kinds of figures, and distributions. Students not only needed to understand procedures to generate and read out the display, but also should be able to criticize them. Meanwhile, for given purpose, students should be able to seize different display advantages and disadvantages. The explanation of data usually resulted in the finding and confirmation of the correlation among data. This relationship might have different levels of complexity and simple linear relationship might easier to be found than reciprocal relation or interaction (NRC, 2008).

In the scientific classroom, when students were conducting scientific exploration and exchange, the investigations of questions as the following might have chances to be contacted to data modeling process (NRC, 1996):

1. What kind of data can be used to answer such question?

2. What were the best observation and measurement?

3. If you want to explain clearly on the solution of the question, how should you organize the data?

4. If you want to make the most convincing explanation, how should you organize the data?

However, in the inquiry-based teaching, this part was only one of many compositions. The data modeling process and characteristic cannot be displayed and students cannot be incubated with data modeling ability. For example, data collection usually needs the use of tools. Students use tools to measure thing that students felt interesting, so as to gain data and students can only have weak confidence on correlation between both of them (NRC, 2008). Usually, when students were explaining the data, they cannot seize the meaning of the data and 
use them as the evidence of hypothesis. In addition, students cannot explain the meaning of the figure (Roberts Gott, \& Glaesser, 2010). Students can use subjective way to add regularity in the data (Gott \& Duggan, 2003). Furthermore, students usually tended to think that new question can only be solved by new data and they rarely think about searching the present data to explore question that was not thought about when students were collecting data at the moment (NRC, 2008). In addition, Lehrer and Schauble (2007) pointed out that students usually have difficulty in giving data consistent structure and they usually neglect the need to include important message displayed by the data, including redundant message. We should provide chance for students to structuralize and display data, then they select, analyze, and revise their creation, which is a very important way for solving the above difficulties.

If scientific teaching can help students to put more attention on how data were generated and on the analysis of data, then they can better understand the data and use the data to do the following works:

1. Describe object, creature, and event;

2. Follow the features to conduct classification;

3. Reason, predict, and find out the correlation;

4. Explain the reason and cause;

5. Acquire the learning growth for doing these works and important goal of science education (NRC, 2007).

According to the above research background, this study aimed at designing teaching model that can promote students' data modeling ability and investigating the effectiveness of teaching model.

\section{Research Design}

This study was quasi-experiment design, which ninth graders were divided into four classes and two groups for teaching. The experiment group will be conducted with data modeling teaching designed by this study, while the control group will be conducted with lecture instruction based on the textbook. The teaching content was the Newton's second law of motion and both groups were conducted with teaching activity of three sessions of classes of 135 minutes. For both groups, before the teaching treatment, test of mechanical concept was implemented as pre-test. After the end of teaching activity, test of data modeling ability of "Force and Motion" and achievement test of "Force and Motion" were used as post-test.

\section{Subject}

In this study, four classes of ninth graders in a junior high school of Kaohsiung were used as research target and two classes respectively for the experiment group and the control group. When students were admitted to the school, classes were divided based on normal distribution. To ensure equal performance of natural science of two groups students before the teaching, the class average score of the previous sectional exam was referred to as the assignment for the experiment group and the control group. There were 46 students in the experiment group and 51 students in the control group. The experiment group was taught by teacher $\mathrm{A}$ and the control group was taught by teacher B, but both of teachers A and B have almost the same teaching experiences.

\section{Treatment}

This study has referred to the structure developed based on the model proposed by Lesh and Doerr (2003) 
to design data modeling teaching for the experiment group. It can be divided into four parts, such as model eliciting, model exploration, model application, and discussion activity.

\section{Model Eliciting Activity}

When conducting this teaching activity, it was needed to find out key variables for the phenomenon to be investigated. Take the "Force and Motion" unit as an example, the major key variable was force exerted, mass, and acceleration. When phenomenon in the daily life related to these three variables was displayed through film or animation, question was proposed regarding key variable in the film. Different thoughts among students were triggered and students' concept was confirmed. Meanwhile, students were answering the question and conducting discussion, students' response was introduced to key variable. Therefore, students can focus on variables to be investigated in model exploration activity to facilitate the subsequent model exploration activity.

\section{Model Exploration Activity}

Students in this activity operate data modeling software to conduct virtual experiment. Moreover, the investigation of "Force and Motion" by this software followed the below steps:

1. Making observation;

2. Asking questions;

3. Selecting and generating variables;

4. Predicting the results of the experiment;

5. Conducting the virtual experiment;

6. Displaying the table and data model;

7. Explaining the meaning of the data model.

8. Comparing with prediction;

9. Drawing conclusion.

Among all the above steps, this software was embedded with reminding scaffolding to guide the students to conduct data modeling exploration. Through students' operation on the software, student can experience data modeling process and software can record problems existed in each step during students' learning process on data modeling. Collection of students' learning problems can facilitate the subsequent discussion activity.

\section{Model Application Activity}

It lets students apply model constructed by them to other situation to generate learning transfer effect.

\section{Discussion Activity}

In such activity, problems were designed based on the learning goal to be reached in each step of data modeling software by the students. Therefore, students can have discussion to deepen the learning result. Next, discussion and clarification were made on the learning problems that might exist in the data modeling process for the students.

\section{Instrument}

\section{Data Modeling Ability Test of "Force and Motion"}

"Force and Motion" data modeling ability test developed by this study has referred to test of graphing in 
science of constructing of the strategic development of constructing data graphs proposed by McKenzie and Padilla (1986). The assessment tool required student to follow the situation, to set up data model, and to explain the meaning of data model. Meanwhile, data modeling ability was divided into "scientific graph construction" and "scientific graph explanation." This study has followed the data modeling steps to divide data modeling ability into three parts, such as "organizing data model," "explaining data model," and "applying data model." The two-way list of "Force and Motion" data modeling ability test is as shown in Table 1.

After checking by expert on "Force and Motion" data modeling ability test developed by this study, pre-test was then conducted. The pre-test targets were from the junior high school in the metropolitan area, junior high school in the suburban area, and remote area of Kaohsiung. Two classes were taken for each junior high school and it comes to a total of 148 ninth graders. The value of Kuder-Richardson (KR) 20 in "Force and Motion” data modeling ability test was 0.878 . After the pre-test was implemented for one month, re-test was conducted and the reliability was 0.714 , which meant that it has good reliability. This test contained 21 problems, including choice and non-choice problems with one point being given to each problem.

Table 1

Two-Way List of "Force and Motion" Data Modeling Ability Test

\begin{tabular}{lll}
\hline & Data modeling ability & Problem No. \\
\hline & Student can judge independent variable and dependent variable in the experiment. & $3(2), 5(3)$ \\
& Student can judge the location of independent variable and dependent variable in the coordinate axis. & $6(1)$ \\
Organizing & Student can follow the experiment content to select correct data model. & $3(3)$ \\
data model & Student can follow experiment data and table to select correct data model. & 1 \\
& Student can follow data to select the most appropriate data model. & $3(1), 4(1)$ \\
& Student can follow experiment data and table to make data model. & $6(2), 6(3)$ \\
Explaining & Student can explain correlation among variables in data model. & $4(3), 5(4)$ \\
data model & Student can explain the meaning of multiple straight lines in data model. & $6(4)$ \\
& Student can explain the correlation between two related data models. & $4(2), 4(5)$ \\
& Given one independent variable value, student can judge from data model corresponding dependent & $3(4)$ \\
Applying & variable value. & $2(3), 4(4)$ \\
data model & Given one set of new experiment data, student can judge from data model the location of the & $5(1), 2(1)$ \\
& experiment result. & $5(2), 2(2)$ \\
\hline
\end{tabular}

\section{"Force and Motion” Achievement Test}

The "Force and Motion" achievement test used in this study was selected from comprehensive assessment program for junior high school students of each year and problems related to the second law of Newton was selected. There are 10 problems with one point being given for each correct answer. After the problem has been reviewed by expert, the research target will then be tested.

\section{“Force and Motion” Data Modeling Software Design}

The "Force and Motion" data modeling software used in this study was interactive simulation software and its major content was to investigate the motion status change after the object was exerted with force. Furthermore, the correlation among force exerted, mass, and acceleration was investigated. This "Force and Motion” data modeling software followed data modeling flow to propose target problem, generate and select variable, select data, organize data model, explain data model, and apply data model, which was sub-ability of data modeling and it was used as basis for the design of interactive simulation software script. Meanwhile, the 
interactive simulation software was finally designed with summative assessment script, so that students can experience and be tested with data modeling ability during the assessment. After finishing the script design, it was co-discussed and revised by two professors in the science education field. Two teachers in physics in senior high school and four teachers in the physics and chemistry in the junior high school, then it was sent to professional software developing company for preparation. After software is set up, it was tested by three junior high school students and parts that will affect student's learning in the software were removed. In this study, "Force and Motion" data modeling software was used as model eliciting, model exploration, and model application activities in the teaching design.

Figure 1 was a mapping between each data modeling ability and "Force and Motion" data modeling software design content. In the "Force and Motion" unit, in order to let students experience reasonable data modeling process so as to set up complete data model, students must experience completely, propose target problem, generate and select variable, and experience the process of data selection and organization of data model. Next, the meaning of the setup data model should be explained, and then, it could be applied to new situation.

As shown in Figure 1, "Force and Motion" data modeling software included:

1. Students can use situation animation to display living situation to be used as model triggering activity and let students focus on the discovery of problems that can be investigated in the situation;

2. Students followed the phenomenon displayed by the situation animation to select problems to be investigated;

3. Students followed the previous step to select correct problem to be investigated, then each variable for experiment was dragged respectively to the location of independent, control, and dependent variable;

4. Students needed to select appropriate independent variable value to measure the result of dependent variable. When drawing a correlation chart, it will need at least five different data points to draw a good experiment result curve and there should be certain interval among these data points;

5. Select appropriate table, then place correctly independent variable and dependent variable into the table. Usually, the independent variable was placed on the first row and dependent variable was placed on the second row;

6. Students can use the pattern of the correlation chart drawn by experiment data to explain the correlation among variables;

7. Students will then investigate sequentially the correlation between mass and acceleration (fixed force exerted) as well as the correlation between force exerted and acceleration (mass is fixed). After integration, the obtained data model can be represented by a mathematical model $F=m a$;

8. At the end of the software, summative assessment of six small questions was designed to be used as activity for applying data model and let students generate learning transfer.

In this study, by summarizing the above contents, "Force and Motion" data modeling learning software was designed and it included interactive process, virtual lab, the providing of real time feedback and scaffolding, and summative assessment, etc.. 


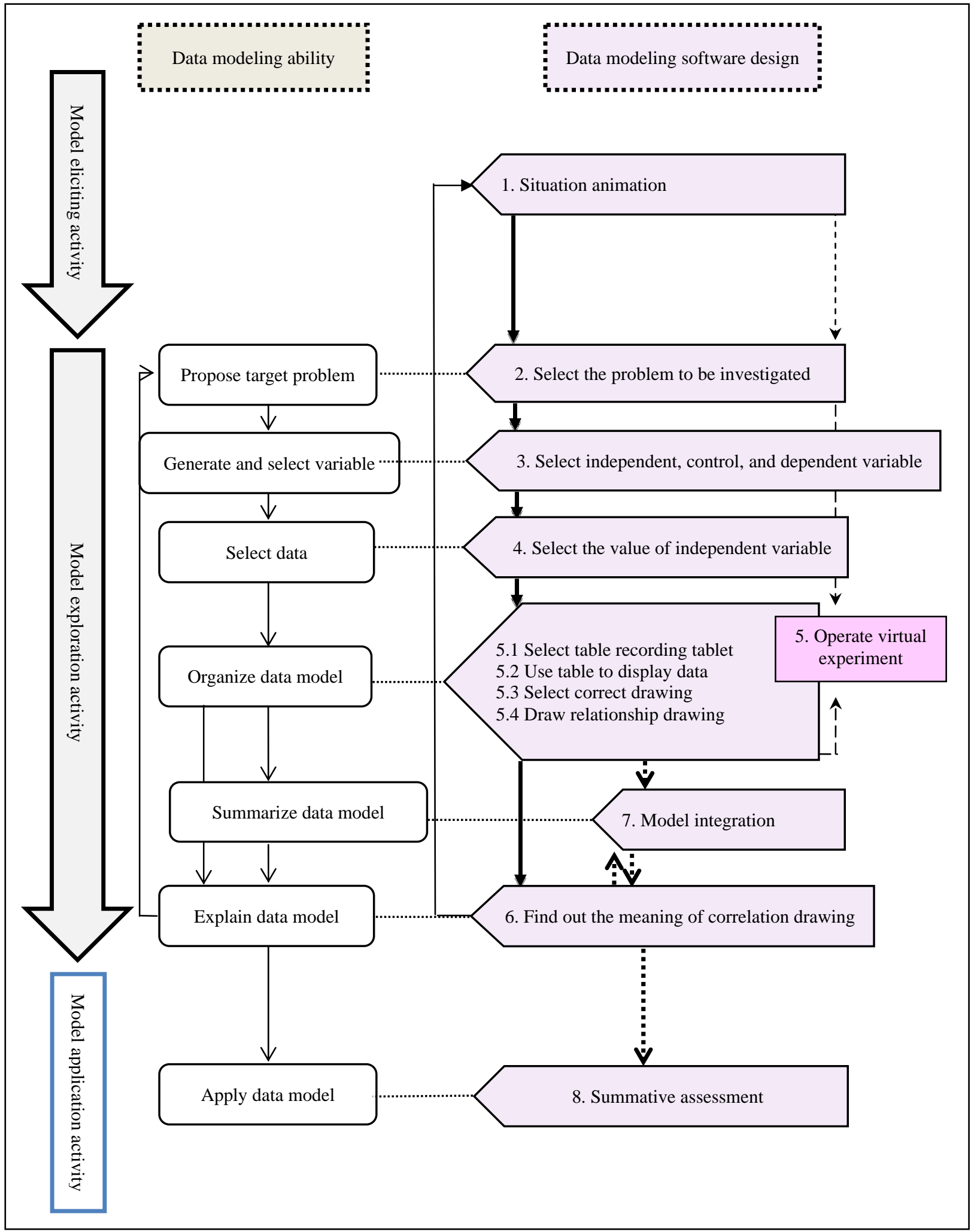

Figure 1. Mapping chart between each data modeling ability and "Force and Motion" data modeling software design content. (Source: Mapping relationship was connected by dotted line.) 


\section{Data Analyses}

In order to compare the performance of data modeling teaching in the experiment group and lecture instruction of "Force and Motion" data modeling ability test and "Force and Motion" learning achievement test in the control group, this study has used the test of mechanical concept before teaching treatment as covariate variable. Students' performance in "Force and Motion" data modeling ability test and "Force and Motion" learning achievement test were used respectively as dependent variable to conduct statistical analysis with analysis of covariance (ANCOVA) so as to remove. Before teaching, the influence of students' understanding on the mechanical concept on the performance of "Force and Motion" data modeling ability test and "Force and Motion" learning achievement test. The significance level of each statistical test in this study was set up as $\alpha=$ 0.05. Furthermore, in order to investigate further the effect of experiment treatment, the experiment effect indicator $\eta^{2}$ of analysis of variance (ANOVA) proposed by Cohen (1988) was used in this study, and they represented the values of large, middle, and small effect size (ES), which were 0.14, 0.06, and 0.01 respectively.

In addition, through analyzing students' average correct answering rate of questions in organizing data model, explaining and applying data model in "Force and Motion" data modeling ability test, following the sectional ability indicator of organizing data model, and explaining and applying data model to collect correct answering rate of ability indicator, problems existed in each data modeling ability performance of students in the experiment group and the control group can be understood and average correct answering rate was used to conduct scoring. Whether students possessed these data modeling abilities should be decided according to learning level instead of relative location within the class. Robert and Miller (2005) pointed out that if the ability indicator of the courses has been clearly explained. If standard of mastery has been set up, then the level of achieving the goal can be used for the definition and it was as in the following descriptions:

A = “Correct answering rate is 95\%-100\%." Students have mastery all the primary and secondary learning targets in the courses;

$\mathrm{B}=$ "Correct answering rate is $85 \%-94 \%$." Students have mastery on major and most of the sub-targets;

C = "Correct answering rate is $75 \%-84 \%$." Students have mastery on the primary learning targets in the courses, but have mastery only on some secondary targets;

$\mathrm{D}=$ "Correct answering rate is $65 \%-74 \%$." Students have only mastery on some primary and secondary teaching targets. They almost did not possess the needed elements for next teaching level and it is better to make remedial teaching;

$\mathrm{F}=$ "Correct answering rate is below 65\%." Students did not have mastery any teaching target in the courses, and important element needed for the next teaching level was also insufficient, which was needed to make remedial teaching.

Therefore, in this study, "F" rank was selected as assessment standard for the problems that might exist in students' data modeling ability. If the question correct answering rate of each ability indicator in the experiment group or the control group students was lower than $65 \%$, then it can explain that students did not achieve in such ability and remedial teaching should be conducted.

\section{Findings and Discussions}

\section{Comparison of "Force and Motion" Data Modeling Ability Between the Two Groups Students}

The score of test of mechanical concept before teaching treatment was used as covariate variable and students' score in "Force and Motion" data modeling ability test was used as dependent variable to conduct one-way of ANCOVA. For the within-the-group regression coefficient homogeneity test result $(F=1.037, p>$ 
0.05), the significance level was not reached. It meant that the relationship between covariate variable and dependent variable will not be different because of the difference of treatment level of independent variable. That is, the hypothesis of homogeneity of within-the-group regression coefficient of covariate variable was met and the ANCOVA can be continued. The result of ANCOVA was shown in Table $2(F=7.323, p<0.05)$, which meant that after removing the influence the score of test of mechanical concept, the performance of "Force and Motion" data modeling ability test of students in the experiment group and the control group reached significant difference $\left(p<0.05\right.$ ). Furthermore, experiment ES $\eta^{2}=0.072$ (larger than 0.058 ), which reached medium scale experiment effect.

Table 2

ANCOVA Results of the Data Modeling Ability Test of Students in Both Groups

\begin{tabular}{lllrrrrr}
\hline & Source of variance & \multicolumn{1}{c}{$S$} & $d f$ & $M S$ & $F$ & $p$ & $\eta^{2}$ \\
\hline \multirow{2}{*}{ Data modeling } & Inter-group & 133.804 & 1 & 133.804 & \multirow{2}{*}{$7.323^{*}$} & \multirow{2}{*}{0.008} & 0.072 \\
& Error & 452.687 & 94 & 4.816 & & & \\
\hline
\end{tabular}

Note. ${ }^{*} p<0.05$.

Table 3 was the abstract of mean $(M)$, standard deviation $(S D)$, and adjusted mean $(\operatorname{Adj} M)$ in data modeling ability test for the experiment group and the control group students. It showed that after teaching, the experiment group students showed superior performance in data modeling ability test than that of the control group.

Table 3

M, SD, and AdjM in Data Modeling Ability Test in the Experiment Group and the Control Group

\begin{tabular}{llllll}
\hline & Group & $N$ & $M$ & $S D$ & AdjM \\
\hline \multirow{2}{*}{ Data modeling } & Experiment group & 46 & 15.090 & 4.774 & 15.197 \\
& Control group & 51 & 12.940 & 5.006 & 12.842 \\
\hline
\end{tabular}

\section{Comparison of "Force and Motion" Achievement Test Between the Two Groups Students}

The score of test of mechanical concept before teaching treatment was used as covariate variable and students' "Force and Motion" achievement test score was used as dependent variable to conduct ANCOVA. For the within-the-group regression coefficient homogeneity test result $(F=0.056, p>0.05$ ), which meant that significance level was not reached. It meant that the relationship between covariate variable and dependent variable will not be different due to different treatment level of independent variable. The hypothesis of within-the-group regression coefficient homogeneity of covariate variable was met, and the ANCOVA can be conducted continuously. The result of ANCOVA was shown in Table $4(F=0.289, p>0.05)$, which meant that the significance level was not reached and it showed that after teaching treatment. Students in the experiment group and the control group did not show significant difference in the performance of "Force and Motion" achievement test.

Table 4

ANCOVA Results of the "Force and Motion" Achievement Test for the Two Group Students

\begin{tabular}{llrrrrrr}
\hline & Source of variance & \multicolumn{1}{c}{$S S$} & $d f$ & $M S$ & $F$ & $p$ & $\eta^{2}$ \\
\hline \multirow{2}{*}{ Data modeling } & Inter-group & 4.596 & 1 & 4.596 & \multirow{2}{*}{1.139} & 0.289 & 0.012 \\
& Error & 379.290 & 94 & 4.035 & & & \\
\hline
\end{tabular}


Table 5 was the abstract $M$, SD, and $A d j M$ in "Force and Motion" achievement test for the experiment group and the control group students.

Table 5

M, SD, and AdjM of "Force and Motion" Achievement Test

\begin{tabular}{llllll}
\hline & Group & $N$ & $M$ & $S D$ & AdjM \\
\hline "Force and Motion” & Experiment group & 46 & 4.200 & 2.197 & 4.250 \\
achievement test & Control group & 51 & 3.860 & 2.450 & 3.814 \\
\hline
\end{tabular}

\section{Learning Problems Existed in the Performance of Data Modeling Ability for the Two Groups Students}

Table 6 was the abstract of average correct answering rate in "Force and Motion" data modeling ability, and it showed the situation of correct questions answering rate in three dimensions of organizing data model, explaining data model, and applying data model for students in the experiment group and the control group. This can be used to understand learning problem existed in data modeling ability for students.

Table 6

Average Correct Answering Rate in Questions of Three Dimensions of "Force and Motion" Data Modeling Ability for Two Groups Students

\begin{tabular}{lll}
\hline Data modeling ability & Group & Average answering rate \\
\hline \multirow{2}{*}{ Organizing data model } & Experiment group & 0.75 \\
& Control group & $0.62^{*}$ \\
Explaining data model & Experiment group & $0.51^{*}$ \\
& Control group & $0.43^{*}$ \\
Applying data model & Experiment group & 0.77 \\
\hline
\end{tabular}

Note. * Remedial teaching was needed.

From Table 6, it can be seen that in organizing data model ability, average correct answering rate for questions in the experiment group was 0.75, while it was 0.62 for the control group (smaller than 65\%). According to the above definition of Robert and Miller (2005), it showed that on such ability, students in the control group needed to be conducted with remedial teaching. In the ability of explaining data model, the average correct answering rate for questions was 0.51 for the experiment group and it was 0.43 for the control group. Average correct answering rate of questions for two groups students was smaller than 65\%, which showed that remedial teaching was needed for two groups students in this ability. In the ability of applying data model, average correct answering rate in the experiment group was 0.77, while the control group was 0.70 . Average correct answering rates for questions for both groups students were all larger than 0.65 and remedial teaching was not needed.

In order to understand further learning problems existed in data modeling ability for students in the experiment group and the control group, according to the previously stated double way list, the average correct answering rate of questions in each ability indicator for two groups students were analyzed and the result was shown in Table 7. 
Table 7

\section{Average Correct Answering Rate of Questions in Each Data Modeling Ability Indicator}

\begin{tabular}{|c|c|c|c|}
\hline & Ability indicator & Average correct an & ering rate \\
\hline \multirow{11}{*}{$\begin{array}{l}\text { Organizing } \\
\text { data model }\end{array}$} & $\begin{array}{l}\text { Students can judge independent variable and dependent variable in the } \\
\text { experiment. }\end{array}$ & $\begin{array}{l}\text { Experiment group } \\
\text { Control group }\end{array}$ & $\begin{array}{l}0.498^{*} \\
0.392^{*}\end{array}$ \\
\hline & \multirow{2}{*}{$\begin{array}{l}\text { Student can judge the location of independent variable and dependent variable } \\
\text { in coordinate axis. }\end{array}$} & Experiment group & 0.663 \\
\hline & & Control group & $0.451^{*}$ \\
\hline & \multirow{2}{*}{ Students can follow the experiment content to select correct data model. } & Experiment group & 0.890 \\
\hline & & Control group & 0.690 \\
\hline & \multirow{2}{*}{ Students can follow experiment data and table to select correct data model. } & Experiment group & 0.960 \\
\hline & & Control group & 0.860 \\
\hline & \multirow{2}{*}{ Students can follow data to select the data model with best fit. } & Experiment group & 0.815 \\
\hline & & Control group & 0.745 \\
\hline & \multirow{2}{*}{ Students can follow experiment data and table to draw data model. } & Experiment group & 0.837 \\
\hline & & Control group & 0.735 \\
\hline \multirow{6}{*}{$\begin{array}{l}\text { Explaining } \\
\text { data model }\end{array}$} & \multirow{2}{*}{ Students can explain correlation among variables in the data model. } & Experiment group & $0.536^{*}$ \\
\hline & & Control group & $0.412^{*}$ \\
\hline & \multirow{3}{*}{ Students can explain the meanings of several straight lines in data model. } & Experiment group & $0.467^{*}$ \\
\hline & & Control group & $0.451^{*}$ \\
\hline & & Experiment group & $0.500^{*}$ \\
\hline & Students can explain the relationship between two related data models. & Control group & $0.430^{*}$ \\
\hline \multirow{6}{*}{$\begin{array}{l}\text { Applying data } \\
\text { model }\end{array}$} & \multirow{2}{*}{$\begin{array}{l}\text { Given an independent variable value, student can use data model to judge } \\
\text { corresponding dependent variable value. }\end{array}$} & Experiment group & 0.652 \\
\hline & & Control group & $0.637^{*}$ \\
\hline & \multirow{2}{*}{$\begin{array}{l}\text { Given one set of new experiment data, students can judge the locations of } \\
\text { experiment results from data model. }\end{array}$} & Experiment group & 0.837 \\
\hline & & Control group & 0.775 \\
\hline & \multirow{2}{*}{$\begin{array}{l}\text { Students can follow the trend of data model to use interpolation or extrapolation } \\
\text { for prediction. }\end{array}$} & Experiment group & 0.804 \\
\hline & & Control group & 0.676 \\
\hline
\end{tabular}

Note. ${ }^{*}$ Remedial teaching was necessary.

From Table 7, it can be seen that among all the ability indicators of organizing data model:

1. In the ability indicator of "students can judge independent variable and dependent variable in the experiment," the average correct answering rate of questions in the experiment group was 0.498 and the average correct answering rate of questions in the control group was 0.392. Both students in the experiment group and the control group did not reach such ability indicator and remedial teaching should be done for both groups.

2. In ability indicator of "students can judge the locations of independent variable and dependent variable in coordinate axis," the average correct answering rate in the control group was 0.451 , while students in the control group should take remedial teaching.

In all ability indicators of explaining data model:

1. Inability indicator of "students can explain the relationship among variables in data model," the average correct answering rate of questions in the experiment group was 0.536, and it was 0.412 in the control group. Students in the experiment group and the control group all cannot reach such ability indicator and remedial teaching should all be done on them.

2. In the ability indicator of "students can explain the meaning of multiple straight lines in data model," the average correct answering rate for questions in the experiment group was 0.467 and the average correct answering rate in the control group was 0.451 . Students in both the experiment group and the control group all 
did not reach such ability indicator and remedial teaching was all needed.

3. In the ability indicator of "students can explain the relationship between two related data models," the average correct answering rate in the experiment group was 0.500 , and it was 0.430 in the control group. Both students in the experiment group and the control group did not reach such ability indicator and remedial teaching was all needed.

Finally, among all the ability indicators of applying data model, in ability indicator of "given one independent variable value, from data model, students can judge corresponding dependent variable value," the average correct answering rate in the control group was 0.637 . Therefore, students in the control group needed to receive remedial teaching.

\section{Discussion}

Lawson (1995) pointed out that among 10 most common reasons for scientific teacher not to use inquiry-based teaching, one of them was that scientific teachers worried about if inquiry-based teaching was used, they were not sure whether students' learning achievement can be maintained or not. In this study, the experiment group was conducted with data modeling teaching. Students' performance in "Force and Motion" achievement test, as compared to that of the control group, which significant different was not reached. It meant that as compared to lecture instruction that most teachers were good at, data modeling teacher can let students maintain the same learning achievement and teachers did not have to worry about such problem. Furthermore, since the performance of students in the experiment group in "Force and Motion" data modeling ability test was superior to that of students in the control group, and medium experiment effect was achieved, which showed that data modeling teaching of the experiment group had teaching practice value than that of lecture instruction.

In the ability of explaining data model, students in the experiment group and the control group showed bad performance of "explaining the relationship among variables in data model," "explaining the meaning of multiple straight lines in data model," and "explaining the correlation between two related data models." When this result was compared to the result made by Onwu (1993) for the research regarding senior high school students' ability to draw graphs, he found that students were most difficult in interrelating the results of two or more graphs and the secondary difficult one was stating relationships. Moreover, this study found that the learning problems existed in the experiment group and the control group students was consistent with the finding of Onwu's study. Why data modeling teaching designed by this study cannot enhance students' ability to explain data model? It was guessed to be due to short three sessions of teaching activities conducted, in other words, the teaching treatment time was not long enough. This part needed further investigation.

\section{Conclusions and Implications}

Data modeling teaching designed by this study included four parts, such as model eliciting, model exploration, model application, and discussion activity. In the first three parts, designed data modeling software was used to conduct virtual experiment. Within it, reminding scaffolding was embedded to guide students to conduct data modeling exploration. Later on, aiming at the data modeling processes, problems were designed to let students have discussion to deepen the learning. Meanwhile, for learning problems that might exist among students, class discussion was conducted for clarification. Although the teaching treatment schedule for this study contained only three sessions of classes, it could still be found that its effect in enhancing data modeling ability was better than the lecture instruction based on textbook and there was no difference in the performance of learning achievement, which can remove teacher's worry. Next, because it had medium experiment ES, it 
was data modeling teaching worth further promotion. Although the experiment group students, the same as that of the control group students, had bad performance in ability for explaining data model, yet the reason behind it needed further study and confirmation. Furthermore, "Force and Motion" data modeling ability test developed by this study only contained three parts of organizing, explaining, and applying data model. It did not include abilities, such as the proposition of target problem, generation and selection of variable, and data selection. In future, in the revision of tool of data modeling ability test, questions of such parts can be added, so that the test tools can assess students' data modeling ability more comprehensively.

\section{References}

Cohen, J. (1988). Statistical power analysis for the behavioral sciences (2nd ed.). Hillsdale, N.J.: Lawrence Earlbaum Associates. English, L. D. (2011). Data modelling in the beginning school years. In P. Sullivan \& M. Goos (Eds.), Proceedings of the 34th Annual Conference of the Mathematics Education Research Group of Australia (pp. 226-234). Alice Springs, N.T.: MERGA Inc..

Gott, R., \& Duggan, S. (2003). Understanding and using scientific evidence: How to critically evaluate data. London: SAGE Publications.

Lawson, A. E. (1995). Science teaching and the development of thinking. Belmont, C.A.: Wadsworth.

Lehrer, R., \& Lesh, R. (2003). Mathematical learning. In W. Reynolds \& G. Miller (Eds.), Comprehensive handbook of psychology (Vol. 7, pp. 357-390). New York, N.Y.: John Wiley.

Lehrer, R., \& Schauble, L. (2000). Modeling in mathematics and science. In R. Glaser (Ed.), Advances in instructional psychology (Vol. 5, pp. 101-159). Mahwah, N.J.: Lawrence Erlbaum Associates Publisher.

Lehrer, R., \& Schauble, L. (2007). Contrasting emerging conceptions of distribution in contexts of error and natural variation. In M. Lovett \& P. Shah (Eds.), Thinking with data (pp. 149-176). Mahwah, N.J.: Erlbaum.

Lesh, R., \& Doerr, H. (2003). Foundation of a models and modeling perspective on mathematics teaching and learning. In R. A. Lesh \& H. Doerr (Eds.), Beyond constructivism: A models and modeling perspective on mathematics teaching, learning, and problem solving (pp. 9-34). Mahwah, N.J.: Lawrence Erlbaum.

McKenzie, D. L., \& Padilla, M. J. (1986). The construction and validation of the test of graphing in science (TOGS). Journal of Research in Science Teaching, 23(7), 571-579.

National Research Council (NRC). (1996). The National Science Education Standards. Washington, D.C.: National Academy Press.

NRC. (2007). Taking science to school: Learning and teaching science in grades K-8. Washington, D.C.: National Academy Press.

NRC. (2008). Ready, set, science: Putting research to work in K-8 science classrooms. Washington, D.C.: National Academies Press.

Onwu, G. O. (1993). Line graphing ability of some Nigerian secondary science students. International Journal of Mathematical Education in Science and Technology, 24(3), 385-390.

Organisation for Economic Cooperation and Development (OECD). (2013). PISA 2015 Draft science framework. Retrieved from http://www.oecd.org/pisa/pisaproducts/Draft\%20PISA\%202015\%20Science\%20 Framework\%20.pdf

Robert, L. L., \& Miller, M. D. (2005). Measurement and assessment in teaching (9th ed.). Upper Saddle River, N.J.: Prentice Hall.

Roberts, R., Gott, R. \& Glaesser, J. (2010). Students' approaches to open-ended science investigation: The importance of substantive and procedural understanding. Research Papers in Education, 25(4), 377-407. 